J Neurosurg 120:1048-1054, 2014

(C)AANS, 2014

\title{
Trigeminal neuralgia occurs and recurs in the absence of neurovascular compression
}

\author{
Clinical article
}

\author{
Albert Lee, M.D., Shirley McCartney, Ph.D., Cole Burbidge, B.A., B.S., \\ Ahmed M. Raslan, M.D., and Kim J. Burchiel, M.D. \\ Neurological Surgery, Oregon Health \& Science University, Portland, Oregon
}

\begin{abstract}
Object. Vascular compression of the trigeminal nerve is the most common factor associated with the etiology of trigeminal neuralgia (TN). Microvascular decompression (MVD) has proven to be the most successful and durable surgical approach for this disorder. However, not all patients with TN manifest unequivocal neurovascular compression (NVC). Furthermore, over time patients with an initially successful MVD manifest a relentless rate of TN recurrence.

Methods. The authors performed a retrospective review of cases of TN Type 1 (TN1) or Type 2 (TN2) involving patients 18 years or older who underwent evaluation (and surgery when indicated) at Oregon Health \& Science University between July 2006 and February 2013. Surgical and imaging findings were correlated.

Results. The review identified a total of 257 patients with TN (219 with TN1 and 38 with TN2) who underwent high-resolution MRI and MR angiography with 3D reconstruction of combined images using OsiriX. Imaging data revealed that the occurrence of TN1 and TN2 without NVC was $28.8 \%$ and $18.4 \%$, respectively. A subgroup of 184 patients underwent surgical exploration. Imaging findings were highly correlated with surgical findings, with a sensitivity of $96 \%$ for TN1 and TN2 and a specificity of $90 \%$ for TN1 and $66 \%$ for TN2.

Conclusions. Magnetic resonance imaging detects NVC with a high degree of sensitivity. However, despite a diagnosis of TN1 or TN2, a significant number of patients have no NVC. Trigeminal neuralgia clearly occurs and recurs in the absence of NVC.
\end{abstract}

(http://thejns.org/doi/abs/10.3171/2014.1.JNS131410)

KEY WoRds - trigeminal neuralgia - neurovascular compression • microvascular decompression • internal neurolysis • Type 1 trigeminal neuralgia • Type 2 trigeminal neuralgia - lack of vascular compression • peripheral nerve

I N 1934 Dandy $^{12}$ first proposed that vascular compression of the trigeminal nerve was a cause of trigeminal neuralgia (TN). However, he also pointed out that vascular contact occasionally occurs without the production of TN and that TN may be present in the absence of contact. ${ }^{12}$ Compression of the trigeminal nerve by a blood vessel at or near the root entry zone ${ }^{12,14,21}$ is still thought to be the most common cause for TN. Devor's "ignition theory" of TN relies on a focus of hyperactivity in the ret-

\footnotetext{
Abbreviations used in this paper: BFFE = balanced fast-field echo; DICOM = Digital Imaging and Communications in Medicine; MRA = MR angiography; MVD = microvascular decompression; $\mathrm{NVC}=$ neurovascular compression; OHSU = Oregon Health \& Science University; $\mathrm{TN}$ = trigeminal neuralgia; TN1 = Type $1 \mathrm{TN}$; TN2 = Type 2 TN.
}

rogasserian root, consistent with a focus of demyelination within the nerve possibly caused by vascular compression. The theory states that "nerve injury results in hyperexcitabilty of injured afferents, which results in after discharges large enough to result in non-nociceptive signal being perceived as pain. This leads to wind-up and both peripheral and central sensitization."13 Other etiologies such as multiple sclerosis, ${ }^{20}$ central or peripheral demyelination, ${ }^{30,31}$ root injury, and tumors may be associated with TN. ${ }^{15}$

Vascular compression of the trigeminal nerve is also known to occur in patients who do not have TN, and TN is known to occur in patients who have no vascular compression. In 1989, Adams questioned the role of "microvascular compression" in the surgical treatment of TN, reviewing all the evidence at the time. He made the argument 


\section{Trigeminal neuralgia and neurovascular compression}

that there may be some other process involved. ${ }^{1}$ A review of autopsy studies reveals some degree of contact between the trigeminal nerve and a blood vessel in $90 \%-100 \%$ of patients with TN, but also in 16\%-58\% of patients without TN. ${ }^{16-19}$ In 2009, Miller et al., ${ }^{35}$ evaluated neurovascular compression (NVC) in patients with and without TN and concluded that trigeminal NVC occurred in asymptomatic patients but was more severe and more proximal in patients with TN. A review of the literature reveals that a wide range (4\%-89\%) of TN patients has no demonstrable vascular contact. ${ }^{2-4,12,19,24,26,34}$ For example, Leal et al. ${ }^{26}$ reported no NVC in $9 \%$ of patients by surgical exploration, and imaging revealed no vessel in relation to the nerve in $12 \%$ of patients. There are also reports in which all patients have NVC. ${ }^{40}$ Improvements in imaging technology resolution capabilities have further reinforced that $\mathrm{TN}$ can occur in the absence of vascular contact. $9,26,34$

TN patients often experience a recurrence after an initially successful surgical procedure. A review of the literature reveals a wide range of recurrence rates of approximately $6 \%-41 \% .5,6,8,10,11,22,23,25,27-29,33,36-39,41-44$ Surgical alternatives after recurrence include repeat exploration for recurrent vascular compression and microvascular decompression (MVD), internal neurolysis, or radiofrequency lesioning. Other alternatives for recurrent TN include partial or complete sensory rhizotomy, balloon rhizotomy, glycerol injections, and radiosurgery. Most experienced surgeons tend to favor less destructive approaches since anesthesia dolorosa (deafferentation pain) is a known, and feared, complication of application of destructive procedures to the trigeminal nerve.

In this study we sought to evaluate, in an independent manner, the presence of NVC in a preoperative TN patient population using 3D-reconstructed balanced fastfield echo (BFFE) images fused with 3D time-of-flight MR angiography (MRA) and 3D Gd-enhanced images. We also sought to determine if $\mathrm{TN}$ recurred in the absence of NVC.

\section{Methods}

\section{Patients}

A retrospective review of patients 18 years of age or older with TN Type 1 (TN1) or Type 2 (TN2) ${ }^{7}$ at Oregon Health \& Science University (OHSU) from July 2006 to February 2013 was undertaken. The study was approved by the OHSU Institutional Review Board.

\section{Imaging}

Patients underwent preoperative high-resolution MRI and MR angiography (MRA) with 3D reconstructions using OsiriX. Magnetic resonance imaging (BFFE and MRA) using a 3-T MR scanner (Philips Achieva) was performed as previously described. ${ }^{34,35}$ The $3 \mathrm{D}$ reconstructions were independently reviewed by a neurosurgical fellow blinded to the surgical findings.

\section{D Image Reconstruction}

All BFFE and MRA images were postprocessed using an OsiriX 32-bit Advanced Open Source Workstation
DICOM viewer (www.osirix-viewer.com) as previously described..$^{34,35}$

\section{Surgical Treatment}

In patients in whom imaging confirmed NVC an MVD was performed. When there was no compression on preoperative imaging, an internal neurolysis procedure, a percutaneous procedure (radiofrequency lesioning), or no surgery was performed. ${ }^{36}$

\section{Surgical Assessment of Vascular Compression}

Video images of the intraoperative microscopic view were compared with the preoperative MRI reconstructions with special attention to the identity and nature of the offending vessel. The neurosurgical fellow determined the presence (yes, no) and side (left, right, bilateral) of compression, and the nature/identity (artery, vein, none) of the offending vessel based on the microscope videos and the $3 \mathrm{D}$ reconstructed images.

\section{Statistical Analysis}

Statistical analysis was performed using SPSS version 16.0 for Windows (SPSS, Inc.). The chi-square test was used for contingency tables with a $p$ value of $<0.05$ considered significant.

\section{Results}

\section{Patient Demographics}

Our review identified 257 patients with TN1 (219 patients) and TN2 (38 patients) who underwent high-resolution MRI/MRA with 3D reconstruction of combined images. In the TN1 group, the patients' mean age was 56 \pm 14.2 years, 133 were female $(60.73 \%), 109$ had rightsided symptoms (49.77\%), and 6 had bilateral symptoms $(2.74 \%)$. In the TN2 group, the patients' mean age was $53.8 \pm 11.7$ years, 27 were female $(71.05 \%), 25$ had rightsided symptoms (65.79\%), and 4 had bilateral symptoms (10.53\%). Review of the medical history revealed that 68 patients had hypertension, 2 patients had multiple sclerosis, 5 patients had a history of cerebrovascular accident, and 13 patients had diabetes mellitus (Table 1).

\section{Surgical History}

Of the 257 patients, 33 (26 in the TN1 group and 7 in the TN2 group) had undergone previous MVD procedures (Table 2). Patients with a history of a prior MVD procedure were evaluated at OHSU, and imaging was performed as described above.

\section{Vascular Compression: Imaging}

Of the 219 TN1 patients, $150(68.49 \%)$ had unilateral compression, 6 (2.74\%) had bilateral compression, and 63 (28.8\%) had no NVC. Compression was due to the superior cerebellar artery in 121 sides (74.7\%), a vein in 20 $(12.4 \%)$, the anterior inferior cerebellar artery in $13(8 \%)$, the vertebral artery in $5(3.1 \%)$, the basilar artery in 2 $(1.2 \%)$, and a tumor in $1(0.62 \%$, meningioma).

Six TN1 patients had bilateral TN1 symptoms at some point in their history. (Note: This refers to the clini- 
TABLE 1: Demographic and clinical characteristics of 257 TN patients*

\begin{tabular}{lcl}
\hline \multicolumn{1}{c}{ Characteristic } & TN1 $(\mathrm{n}=219)$ & TN2 $(\mathrm{n}=38)$ \\
\hline mean age (yrs) & 56 & 53.8 \\
mean postop follow up (mos) & 42 & 62 \\
number of females & $133(60.73)$ & $27(71.05)$ \\
right-sided Sxs & $109(49.77)$ & $25(65.79)$ \\
history of hypertension & $62(28.3)$ & $6(15.8)$ \\
history of CVA & $4(1.9)$ & $1(2.6)$ \\
history of MS & $2(0.9)$ & $0(0)$ \\
history of DM & $12(5.5)$ & $1(2.6)$ \\
\hline
\end{tabular}

* Values represent numbers of patients (\%) unless otherwise indicated. CVA = cerebrovascular accident; $\mathrm{DM}=$ diabetes mellitus; $\mathrm{MS}$ = multiple sclerosis; Sxs = symptoms.

cal syndrome of bilateral TN1. The fact that the number of patients with bilateral symptoms is the same (6) as the number of patients who had bilateral compression on imaging is a coincidence.) Of these 6 patients with bilateral symptoms, 2 patients had bilateral compression, 1 patient had unilateral compression, and 3 patients had no compression, as determined by imaging. Therefore, in patients with bilateral TN1, only 5 of $12(41.67 \%)$ sides had visible NVC on imaging studies.

Of the 38 TN2 patients, $31(81.58 \%)$ had unilateral compression. The offending vessel was the superior cerebellar artery in 26 cases $(68.42 \%)$, a vein in $3(7.89 \%)$, and the anterior inferior cerebellar artery in $2(5.26 \%)$. There were no patients with bilateral NVC, and 7 had no NVC (18.4\%) (Table 3).

No patient with TN2 had bilateral compression (Table 3); however, 4 patients had bilateral TN2 symptoms: 3 of these patients had unilateral compression, and 1 patient had no compression. Therefore, in this small group, only $3(37.5 \%)$ of 8 sides had visible compression on imaging studies.

\section{Vascular Compression: Surgical Confirmation}

Surgical exploration was performed in 184 of 257 patients. Of this subset, 156 patients had TN1 (160 procedures).

Among the TN1 patients who underwent their initial surgery at OHSU (145 procedures), there were 115 cases in which imaging compression predictions were confirmed surgically (true positives). There were 3 false positives, 23 true negatives, and 4 false negatives.

Among the TN1 patients who had initial surgery at an outside hospital and subsequently underwent reexploration surgery at OHSU (15 procedures), there were 9 true positives, no false positives, 5 true negatives, and 1 false negative.

Among the TN2 patients who underwent their initial surgery at OHSU (27 procedures), there were 23 true positives, 1 false positive, 2 true negatives, and 1 false negative. Among the 3 TN2 patients who had initial surgery at an outside hospital, there was 1 true positive, 1 false positive, and 1 true negative (Table 4).
TABLE 2: Previous procedures performed at an outside hospital for TN1 and TN2 patients*

\begin{tabular}{lcc}
\hline \multicolumn{1}{c}{ Procedure } & TN1 $(\mathrm{n}=26)$ & TN2 $(\mathrm{n}=7)$ \\
\hline MVD & 8 & 2 \\
1 procedure & 1 & 1 \\
2 procedures & 1 & 1 \\
partial rhizotomy & 1 & \\
neurectomy & 1 & 1 \\
glycerol injection & & \\
RFL & 1 & 1 \\
1 procedure & 2 & \\
multiple procedures & & \\
Gamma Knife surgery & 4 & \\
1 procedure & 3 & \\
$\quad$ \& MVD & 1 & \\
$\quad$ \& RFL & 1 & \\
$\quad$ \& glycerol injection & 1 & \\
\& balloon rhizotomy & 1 & \\
\& MVD \& RFL &
\end{tabular}

* Values represent numbers of patients. RFL = radiofrequency lesioning.

The sensitivity of imaging as a predictor of NVC for both TN1 and TN2 was $96 \%$. The specificity of imaging findings for patients with TN1 and TN2 were 90\% and $66 \%$, respectively.

\section{Discussion}

As early as 1934 Dandy pointed out that vascular

TABLE 3: Type and source of compression on imaging in 219 patients with TN1 and 38 with TN2*

\begin{tabular}{lcl}
\hline Type or Source of Compression & TN1 & TN2 \\
\hline Type & & \\
unilat compression & $150(68.49)$ & $31(81.58)$ \\
bilat compression & $6(2.74)$ & $0(0)$ \\
no compression & $63(28.77)$ & $7(18.42)$ \\
Source & & \\
SCA & $121(74.69)$ & $26(68.42)$ \\
venous & $20(12.35)$ & $3(7.89)$ \\
AICA & $13(8.02)$ & $2(5.26)$ \\
VA & $5(3.09)$ & $0(0)$ \\
BA & $2(1.23)$ & $0(0)$ \\
tumor & $1(0.62)$ & $0(0)$ \\
no compression & $70(31.11)$ & $7(18.42)$ \\
\hline
\end{tabular}

* Values represent numbers of patients (\%) for type of compression and trigeminal nerves (\%) for source of compression. Because 6 of the patients in the TN1 group had bilateral compression, percentages for source of compression in that group are based on a denominator of 225. AICA = anterior inferior cerebellar artery; BA = basilar artery; SCA = superior cerebellar artery; VA = vertebral artery. 


\section{Trigeminal neuralgia and neurovascular compression}

TABLE 4: Trigeminal neuralgia compression sensitivity and specificity*

\begin{tabular}{|c|c|c|c|c|c|c|c|}
\hline $\begin{array}{c}\text { TN Type \& Procedure } \\
\text { Location }\end{array}$ & True Positives & False Positives & True Negatives & False Negatives & Total & $\begin{array}{c}\text { Sensitivity } \\
(\%)\end{array}$ & $\begin{array}{l}\text { Specificity } \\
(\%)\end{array}$ \\
\hline \multicolumn{8}{|l|}{ TN1 (160 procedures) } \\
\hline OHSU & 115 & 3 & 23 & 4 & 145 & & \\
\hline outside hospital & 9 & 0 & 5 & 1 & 15 & & \\
\hline total & 124 & 3 & 28 & 5 & 160 & 96.12 & 90.32 \\
\hline \multicolumn{8}{|l|}{ TN2 (30 procedures) } \\
\hline OHSU & 23 & 1 & 2 & 1 & 27 & & \\
\hline outside hospital & 1 & 1 & 1 & 0 & 3 & & \\
\hline total & 24 & 2 & 3 & 1 & 30 & 96.00 & 66.00 \\
\hline total TN1 + TN2 & 148 & 5 & 31 & 6 & 190 & 96.11 & 86.11 \\
\hline
\end{tabular}

contact occasionally occurs without the production of TN and that TN may be present in the absence of contact..$^{12}$ In 1982, Adams et al. reported "our failure to be convinced by vascular compression as a cause for the majority of our patients' pain."2

Hamlyn, in 1992, noted that an explanation for TN cases in which no vessel was found in contact with the trigeminal nerve at operation was needed and that it should be possible to identify those cases preoperatively. ${ }^{19}$ In 2009, Miller et al. stated that "trigeminal NVC occurs in asymptomatic patients but is more severe and more proximal in patients with TN." 35 While vascular compression of the trigeminal nerve by a blood vessel at or near the root entry zone $\mathrm{z}^{12,14,21}$ remains the primary pathology of $\mathrm{TN}$, there remains an unexplained subset of cases of TN without clear NVC. Improvements in surgical approaches and advances in imaging technology have only reinforced this discrepancy. ${ }^{2-4,11,15,18,19}$ In the case series presented here, $28.8 \%$ of TN1 and $18.4 \%$ of TN2 patients exhibited no NVC.

Trigeminal neuralgia recurrence rates after an initially successful MVD have been reported as ranging from $6 \%$ to $41 \%$ (Table 5). 5,6,8,10,11,22,23,25,27-29,33,36-39,41-44 Van Loveren et al. reported that of $50 \mathrm{TN}$ patients treated by MVD, 84\% were pain free at 3 years. ${ }^{42}$ Liao et al. found that after 80 MVD procedures there were 5 cases of recurrence within 12 months. ${ }^{29}$ Barker et al. ${ }^{5}$ examined 1185 patients who underwent an MVD for TN over a 20-year period. Patients were followed for 1 year or longer after surgery, with $91 \%$ having at least 5 years' follow-up. The superior cerebellar artery was the cause of compression in $75 \%$ of patients, and the anterior inferior cerebellar artery in $10 \%$. A compressive vein was observed in $68 \%$ of patients, and in $12 \%$ a vein was the sole cause of compression. Recurrence of TN occurred in 282 patients $(23.8 \%)$, and reoperation was performed in 132 patients. The authors concluded that higher rates of recurrence occurred in patients in the following subgroups: 1) in females, 2) in patients with more than 8 years of TN prior to surgery, 3 ) in patients who underwent decompression of a vein dur- ing surgery, and 4) in patients with a lack of immediate postoperative relief.5 Bederson and Wilson reviewed 252 MVDs performed in 246 patients. Thirty patients had no observable compression and were treated with partial sensory rhizotomy, and 56 patients had vascular contact without distortion and were treated with MVD and partial sensory rhizotomy. ${ }^{6}$ Zakrzewska and Thomas evaluated 475 patients with TN. Sixty-five MVD procedures were performed in 55 patients; at 5 years' follow-up, $38 \%$ of the MVD patients had had a TN recurrence. ${ }^{43}$ Rath et al. reported on 135 MVDs with a duration of follow-up ranging from 3 months to 5 years (mean 1.4 years). Venous compression was seen in 9 patients, and 7 patients $(5 \%)$ had no venous or arterial compression. The recurrence rate was $16.7 \%$ at 5 years. The authors also commented that patients who had undergone previous destructive procedures had worse outcomes. ${ }^{36}$ Sun et al. reviewed 61 cases in which patients had undergone MVD for TN with a duration of follow-up ranging from 1.1 to 10.5 years (mean $6.7)$. Of the 61 patients, $10(16 \%)$ had recurrence within 2 years of surgery. ${ }^{38}$ Tronnier et al. reviewed 225 cases in which patients were treated with MVD and found that $63 \%$ of the patients had 20 years of pain relief. ${ }^{41}$ Sindou et al. evaluated 120 patients after MVD, with a mean followup period of 41 months; $83.3 \%$ and $79 \%$ of patients had pain relief from sitting craniotomy and lateral approach, respectively. ${ }^{37}$ Cutbush and Atkinson evaluated 109 cases in which MVD was performed by a single surgeon. The mean duration of follow-up was 4.8 years, and 83 patients (76\%) had resolution of their pain. The authors commented that most $(66 \%)$ of the recurrences occurred within 12 months and the superior cerebellar artery was the vessel involved in over $70 \%$ of the cases. ${ }^{11}$ Mendoza and Illingworth evaluated 132 patients who had 133 MVDs (one patient had bilateral symptoms). Follow-up in this series ranged from 6 months to 15 years (mean 5.3 years). After $95(71 \%)$ of the procedures the patients had lasting resolution of their TN, and after 21 (16\%), the patients had a minor recurrence. There was a statistically significant association between operative findings of compression and 
TABLE 5: Summary of lack of compression and recurrence rates for MVD and radiofrequency lesioning procedures

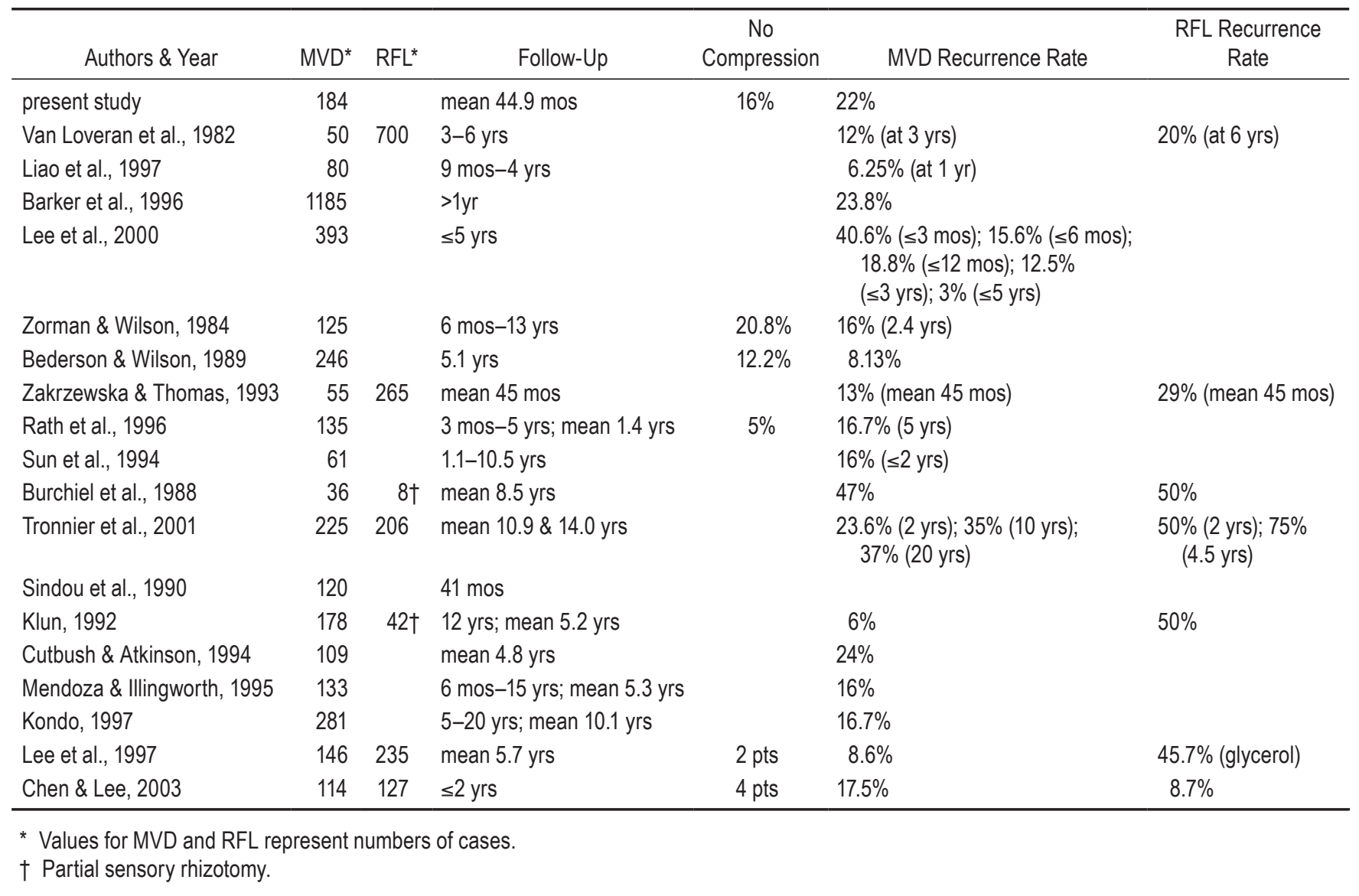

long-term pain relief. ${ }^{33}$ Lee et al. evaluated 116 patients after MVD with 2 years' follow-up and 9 recurrences $(8.6 \%)$. At least 2 patients in their series had no vascular compression and had pain recurrence. ${ }^{27}$ Chen and Lee reviewed 114 cases in which patients had undergone MVD with at least 2 years' follow-up and found a recurrence rate of $17.5 \%$. They noted that 4 patients did not have any visible compression at the time of surgery, and 9 patients had a prominent bone spur or acute angulation at the entry into Meckel's cave as the proposed cause of compression. ${ }^{10}$

Long-term studies demonstrate a less robust outcome for rhizotomy than for MVD in patients with TN. Burchiel et al. showed that after MVD or trigeminal rhizotomy (bipolar cautery of the lateral two-thirds of the trigeminal nerve after crushing) recurrence occurred in $47 \%$ of the MVD group and in 50\% in the trigeminal rhizotomy group with a mean follow-up period of 8.5 years. ${ }^{8}$ Zorman and Wilson evaluated 125 cases in which patients underwent MVD or partial sensory rhizotomies. The mean duration of follow-up was 26 months (range 6 months-13 years). In 26 patients there was no compression seen at the root entry zone, and those patients were treated with partial sensory rhizotomy. The authors reported a 91\% success rate of pain relief. ${ }^{44}$ Klun evaluated 178 patients after MVD and 42 patients after rhizotomy with a follow-up period of up to 12 years (mean 5.2 years). Eleven patients had tumors, 3 patients had multiple sclerosis, and 4 pa- tients had bilateral pain; he excluded patients with "atypical pain." The rate of complete relief after 5 years was $84 \%$ for both operations. ${ }^{23}$

It is important to note that in all series mentioned above there was no differentiation of TN1/TN2 symptomology, no confirmatory preoperative imaging, and no indication of the degree or severity of NVC.

From the studies referenced above, two broad conclusions can be drawn. First, there seems to be a general consensus that some patients with TN do not manifest NVC at the time of surgical exploration. The mechanism for $\mathrm{TN}$ in these cases is unexplained. Second, despite initially effective MVD, there is a steady rate of pain recurrence, which averages $4 \%-5 \%$ per year. $^{2-4,12,19,24,26,34}$ There is no consensus as to the etiology of pain recurrence in these cases.

We believe that the concordance of MRI and operative findings is the most compelling aspect of our results. Furthermore, our results would suggest that high-resolution MRI/MRA can reliably detect NVC in patients with TN. Our imaging indicates that $28.8 \%$ of patients with TN1 do not manifest NVC. Further, post-MVD imaging can discern whether trigeminal NVC has been effectively relieved. The result of out post-MVD imaging studies is that most patients who have recurrent TN after MVD do not have recurrent NVC. The main exception to this finding appears to be when the original NVC was either missed or inadequately decompressed at the time of the first MVD. 


\section{Trigeminal neuralgia and neurovascular compression}

We have previously demonstrated that $17 \%$ of the general population manifests $\mathrm{NVC}$ of the trigeminal nerve. ${ }^{35} \mathrm{If}$, as has been estimated, the incidence of $\mathrm{TN}$ in the population is $1: 10,000(0.01 \%),{ }^{32}$ then $99.94 \%$ of individuals with trigeminal NVC do not have TN. Given these statistics, and the present evidence that TN can both occur and recur without NVC, the hypothesis that TN is caused by neurovascular conflict must be challenged.

\section{Conclusions}

High-resolution MRI has excellent sensitivity and specificity for the pre- and postoperative detection of NVC in TN1. Further, TN1 and TN2 both occur, and recur, in the absence of NVC.

\section{Disclosure}

The authors report no conflict of interest concerning the materials or methods used in this study or the findings specified in this paper.

Author contributions to the study and manuscript preparation include the following. Conception and design: Burchiel. Acquisition of data: Lee, Burbidge. Analysis and interpretation of data: Lee, Burbidge. Drafting the article: Burchiel, Lee, McCartney, Burbidge, Raslan. Critically revising the article: Burchiel, Lee, McCartney, Raslan. Reviewed submitted version of manuscript: all authors. Approved the final version of the manuscript on behalf of all authors: Burchiel. Statistical analysis: Lee, Burbidge. Administrative/techni$\mathrm{cal} /$ material support: McCartney. Study supervision: Burchiel.

\section{References}

1. Adams CB: Microvascular compression: an alternative view and hypothesis. J Neurosurg 70:1-12, 1989

2. Adams CB, Kaye AH, Teddy PJ: The treatment of trigeminal neuralgia by posterior fossa microsurgery. J Neurol Neurosurg Psychiatry 45:1020-1026, 1982

3. Anderson VC, Berryhill PC, Sandquist MA, Ciaverella DP, Nesbit GM, Burchiel KJ: High-resolution three-dimensional magnetic resonance angiography and three-dimensional spoiled gradient-recalled imaging in the evaluation of neurovascular compression in patients with trigeminal neuralgia: a double-blind pilot study. Neurosurgery 58:666-673, 2006

4. Apfelbaum RI: Surgery for tic douloureux. Clin Neurosurg 31:351-368, 1983

5. Barker FG II, Jannetta PJ, Bissonette DJ, Larkins MV, Jho HD: The long-term outcome of microvascular decompression for trigeminal neuralgia. N Engl J Med 334:1077-1083, 1996

6. Bederson JB, Wilson CB: Evaluation of microvascular decompression and partial sensory rhizotomy in 252 cases of trigeminal neuralgia. J Neurosurg 71:359-367, 1989

7. Burchiel KJ: A new classification for facial pain. Neurosurgery 53:1164-1167, 2003

8. Burchiel KJ, Clarke H, Haglund M, Loeser JD: Long-term efficacy of microvascular decompression in trigeminal neuralgia. J Neurosurg 69:35-38, 1988

9. Cha J, Kim ST, Kim HJ, Choi JW, Kim HJ, Jeon P, et al: Trigeminal neuralgia: assessment with T2 VISTA and FLAIR VISTA fusion imaging. Eur Radiol 21:2633-2639, 2011

10. Chen JF, Lee ST: Comparison of percutaneous trigeminal ganglion compression and microvascular decompression for the management of trigeminal neuralgia. Clin Neurol Neurosurg 105:203-208, 2003

11. Cutbush K, Atkinson RL: Treatment of trigeminal neuralgia by posterior fossa microvascular decompression. Aust $\mathbf{N} \mathbf{Z}$ J Surg 64:173-176, 1994
12. Dandy WE: Concerning the cause of trigeminal neuralgia. Am J Surg 24:447-455, 1934

13. Devor M, Amir R, Rappaport ZH: Pathophysiology of trigeminal neuralgia: the ignition hypothesis. Clin J Pain 18:4-13, 2002

14. Fromm GH, Sessle BJ: Trigeminal Neuralgia: Current Concepts Regarding Pathogenesis and Treatment. Boston: Butterworth-Heinemann, 1991

15. Gronseth G, Cruccu G, Alksne J, Argoff C, Brainin M, Burchiel K, et al: Practice parameter: the diagnostic evaluation and treatment of trigeminal neuralgia (an evidence-based review): report of the Quality Standards Subcommittee of the American Academy of Neurology and the European Federation of Neurological Societies. Neurology 71:1183-1190, 2008

16. Haines SJ, Jannetta PJ, Zorub DS: Microvascular relations of the trigeminal nerve. An anatomical study with clinical correlation. J Neurosurg 52:381-386, 1980

17. Hamlyn PJ: Neurovascular relationships in the posterior cranial fossa, with special reference to trigeminal neuralgia. 1. Review of the literature and development of a new method of vascular injection-filling in cadaveric controls. Clin Anat 10:371-379, 1997

18. Hamlyn PJ: Neurovascular relationships in the posterior cranial fossa, with special reference to trigeminal neuralgia. 2 . Neurovascular compression of the trigeminal nerve in cadaveric controls and patients with trigeminal neuralgia: quantification and influence of method. Clin Anat 10:380-388, 1997

19. Hamlyn PJ, King TT: Neurovascular compression in trigeminal neuralgia: a clinical and anatomical study. J Neurosurg 76: 948-954, 1992

20. Harris W: Rare forms of paroxysmal trigeminal neuralgia, and their relation to disseminated sclerosis. BMJ 2:1015-1019, 1950

21. Jannetta PJ: Arterial compression of the trigeminal nerve at the pons in patients with trigeminal neuralgia. J Neurosurg 26 Suppl:159-162, 1967

22. Kanpolat Y, Savas A, Bekar A, Berk C: Percutaneous controlled radiofrequency trigeminal rhizotomy for the treatment of idiopathic trigeminal neuralgia: 25-year experience with 1,600 patients. Neurosurgery 48:524-534, 2001

23. Klun B: Microvascular decompression and partial sensory rhizotomy in the treatment of trigeminal neuralgia: personal experience with 220 patients. Neurosurgery 30:49-52, 1992

24. Kolluri S, Heros RC: Microvascular decompression for trigeminal neuralgia. A five-year follow-up study. Surg Neurol 22:235-240, 1984

25. Kondo A: Follow-up results of microvascular decompression in trigeminal neuralgia and hemifacial spasm. Neurosurgery 40:46-52, 1997

26. Leal PR, Hermier M, Froment JC, Souza MA, Cristino-Filho G, Sindou M: Preoperative demonstration of the neurovascular compression characteristics with special emphasis on the degree of compression, using high-resolution magnetic resonance imaging: a prospective study, with comparison to surgical findings, in 100 consecutive patients who underwent microvascular decompression for trigeminal neuralgia. Acta Neurochir (Wien) 152:817-825, 2010

27. Lee KH, Chang JW, Park YG, Chung SS: Microvascular decompression and percutaneous rhizotomy in trigeminal neuralgia. Stereotact Funct Neurosurg 68:196-199, 1997

28. Lee SH, Levy EI, Scarrow AM, Kassam A, Jannetta PJ: Recurrent trigeminal neuralgia attributable to veins after microvascular decompression. Neurosurgery 46:356-362, 2000

29. Liao JJ, Cheng WC, Chang CN, Yang JT, Wei KC, Hsu YH, et al: Reoperation for recurrent trigeminal neuralgia after microvascular decompression. Surg Neurol 47:562-570, 1997

30. Love S, Coakham HB: Trigeminal neuralgia: pathology and pathogenesis. Brain 124:2347-2360, 2001

31. Love S, Hilton DA, Coakham HB: Central demyelination of the 
Vth nerve root in trigeminal neuralgia associated with vascular compression. Brain Pathol 8:1-12, 1998

32. Manzoni GC, Torelli P: Epidemiology of typical and atypical craniofacial neuralgias. Neurol Sci 26 (Suppl 2):s65-s67, 2005

33. Mendoza N, Illingworth RD: Trigeminal neuralgia treated by microvascular decompression: a long-term follow-up study. Br J Neurosurg 9:13-19, 1995

34. Miller J, Acar F, Hamilton B, Burchiel K: Preoperative visualization of neurovascular anatomy in trigeminal neuralgia. J Neurosurg 108:477-482, 2008

35. Miller JP, Acar F, Hamilton BE, Burchiel KJ: Radiographic evaluation of trigeminal neurovascular compression in patients with and without trigeminal neuralgia. Clinical article. J Neurosurg 110:627-632, 2009

36. Rath SA, Klein HJ, Richter HP: Findings and long-term results of subsequent operations after failed microvascular decompression for trigeminal neuralgia. Neurosurgery 39:933-940, 1996

37. Sindou M, Amrani F, Mertens P: [Microsurgical vascular decompression in trigeminal neuralgia. Comparison of 2 technical modalities and physiopathologic deductions. A study of 120 cases.] Neurochirurgie 36:16-26, 1990 (Fr)

38. Sun T, Saito S, Nakai O, Ando T: Long-term results of microvascular decompression for trigeminal neuralgia with reference to probability of recurrence. Acta Neurochir (Wien) 126:144-148, 1994

39. Taha JM, Tew JM Jr: Treatment of trigeminal neuralgia by percutaneous radiofrequency rhizotomy. Neurosurg Clin $\mathbf{N}$ Am 8:31-39, 1997
40. Tanaka T, Sakamoto E, Shiiba S, Oda M, Kito S, WakasugiSato N, et al: Relationship between the curative effects of carbamazepine administration and the neurovascular compression volume of the trigeminal nerve measured using magnetic resonance cisternography. Clin J Pain 25:752-759, 2009

41. Tronnier VM, Rasche D, Hamer J, Kienle AL, Kunze S: Treatment of idiopathic trigeminal neuralgia: comparison of longterm outcome after radiofrequency rhizotomy and microvascular decompression. Neurosurgery 48:1261-1268, 2001

42. van Loveren H, Tew JM Jr, Keller JT, Nurre MA: A 10-year experience in the treatment of trigeminal neuralgia. Comparison of percutaneous stereotaxic rhizotomy and posterior fossa exploration. J Neurosurg 57:757-764, 1982

43. Zakrzewska JM, Thomas DG: Patient's assessment of outcome after three surgical procedures for the management of trigeminal neuralgia. Acta Neurochir (Wien) 122:225-230, 1993

44. Zorman G, Wilson CB: Outcome following microsurgical vascular decompression or partial sensory rhizotomy in 125 cases of trigeminal neuralgia. Neurology 34:1362-1365, 1984

Manuscript submitted July 1, 2013

Accepted January 7, 2014.

Please include this information when citing this paper: published online February 7, 2014; DOI: 10.3171/2014.1.JNS131410.

Address correspondence to: Kim J. Burchiel, M.D., Department of Neurological Surgery, Oregon Health \& Science University, mail code: CH8N, 3303 SW Bond Ave., Portland, Oregon 97239-3098. email: burchiek@ohsu.edu. 